\title{
Is the $\mathrm{VO}_{2}$ max that we measure really maximal?
}

\author{
Bruno P. C. Smirmaul ${ }^{1}$, Danilo R. Bertucci ${ }^{1,2}$ and Inaian P. Teixeira1 \\ 1 Department of Physical Education, São Paulo State University (UNESP), Rio Claro, Brazil \\ ${ }^{2}$ Department of Physiological Sciences, Federal University of São Carlos (UFSCAR), São Carlos, Brazil \\ *Correspondence: brunosmirmaul@gmail.com
}

Edited by:

Simon C. Gandevia, Neuroscience Research Australia, Australia

\section{INTRODUCTION}

The maximal oxygen uptake $\left(\mathrm{VO}_{2} \max \right)$ can be defined as the maximum integrated capacity of the pulmonary, cardiovascular and muscular systems to uptake, transport and utilize $\mathrm{O}_{2}$, respectively (Poole et al., 2008). Usually measured by the incremental exercise test in the treadmill or cycle ergometer, the $\mathrm{VO}_{2} \max$ test has become a cornerstone in clinical and applied physiology involving physical exercise. Its applications are numerous, ranging from elite athletes to individuals with several pathologic conditions (Mancini et al., 1991; Bassett and Howley, 2000). Despite studied for approximately a century, questions regarding the $\mathrm{VO}_{2} \max$ are still source of debate and disagreement in the literature (Noakes, 1998; Bergh et al., 2000; Levine, 2008; Ekblom, 2009; Noakes and Marino, 2009; Spurway et al., 2012). In particular, the study of the methods of $\mathrm{VO}_{2} \max$ measurement is a field of investigation that has been challenging through the years (Midgley et al., 2007, 2008). Intriguing findings recently published (Beltrami et al., 2012; Mauger and Sculthorpe, 2012) bring additional debate regarding the measurement of the true $\mathrm{VO}_{2} \max$ value and its limiting/regulatory mechanisms. In this article we briefly describe the current testing methods and mechanisms of $\mathrm{VO}_{2}$ max limitation/regulation, and discuss the new findings of these two recent studies and their possible implications in the field.

\section{CURRENT MEASUREMENT AND $\mathrm{VO}_{2}$ max LIMITING/REGULATORY MECHANISMS}

One of the most popular concepts used to obtain $\mathrm{VO}_{2}$ max during an incremental exercise test is the occurrence of the plateau. The origin of this concept had its basis in the studies of Hill and Lupton (1923) 90 years ago, in which they proposed the existence of an individual exercise intensity beyond which there is no increase in the $\mathrm{VO}_{2}$, representing the limit of the cardiorespiratory capacity. However, the need for the plateau occurrence to the $\mathrm{VO}_{2}$ max determination presents limitations, once it conflicts with the fact that its occurrence is not universal (Doherty et al., 2003; Astorino et al., 2005). With the purpose to solve this problem and ensure that individuals attain always "maximal" conditions by the end of an incremental exercise test, producing true $\mathrm{VO}_{2}$ max values, the use of physiological parameters as criteria for exercise test interruption based upon respiratory exchange ratio, maximal heart rate and blood lactate concentrations became popular (Poole et al., 2008). These parameters, though, when used as criteria for $\mathrm{VO}_{2}$ max determination, can underestimate the actual measured value up to $26 \%$ (Poole et al., 2008). Finally, the current solution proposed to $\mathrm{VO}_{2}$ max determination when the plateau does not occur, is the use of the $\mathrm{VO}_{2}$ peak, which seems to be a consistent $\mathrm{VO}_{2}$ max index, as long as a constant supramaximal exercise test is done after the incremental test, called "verification phase" (Day et al., 2003; Midgley and Carroll, 2009).

Presently, two main theoretical models are discussed in the literature aiming to explain the mechanisms of $\mathrm{VO}_{2}$ max limitation and/or regulation. The classical model proposes that $\mathrm{VO}_{2}$ max is limited by the maximal capacity of the heart to provide $\mathrm{O}_{2}$ to the muscles, that means, when one reaches the $\mathrm{VO}_{2}$ max the cardiovascular system is working on its limit (Ekblom, 2009). Alternatively, the other model advocates that the cardiovascular system never reaches a limit of work, and that $\mathrm{VO}_{2} \max$ is regulated, rather than limited, by the number of motor unit recruited in the exercising limbs, which is always submaximal (Noakes and Marino, 2009). Thus, this model proposes that there is always a physiological reserve, both cardiovascular and neuromuscular, once the number of motor unit recruited by the active muscles during exercise is regulated by the brain to prevent catastrophic failure in bodily systems (Noakes and Marino, 2009).

\section{IS THE $\mathrm{VO}_{2}$ max THAT WE MEASURE REALLY MAXIMAL?}

Independently of the $\mathrm{VO}_{2} \max$ limiting/regulatory mechanisms (Ekblom, 2009; Noakes and Marino, 2009), it is believed that implementing specific criteria during the incremental exercise test as duration (Midgley et al., 2008), presence of the "verification phase" (Day et al., 2003; Midgley and Carroll, 2009), and rate of $\mathrm{VO}_{2}$ sample acquisition (Astorino, 2009), one obtains true $\mathrm{VO}_{2} \max$ values. Two recent studies, however, challenge such beliefs.

The first study (Mauger and Sculthorpe, 2012) compared a conventional incremental exercise test (i.e., with fixed load increments until voluntary exhaustion) with a maximal self-paced incremental exercise test regulated by individual perception of effort. The total duration of the latter was $10 \mathrm{~min}$, distributed in 5 stages of 2 min each, in which individuals controlled the exercise intensity at each moment in order to achieve individual perceptions of effort of $11,13,15,17$, and 20 , respectively, in the 15-points Borg scale. Interestingly, this maximal self-paced incremental test resulted in a significantly higher $\mathrm{VO}_{2}$ max $(\approx 8 \%$; Figure 1A) when compared to the values found during the conventional incremental exercise test (Mauger and Sculthorpe, 2012).

The second study (Beltrami et al., 2012) compared a conventional incremental exercise test with a decremental protocol (i.e., with decreasing exercise intensity 


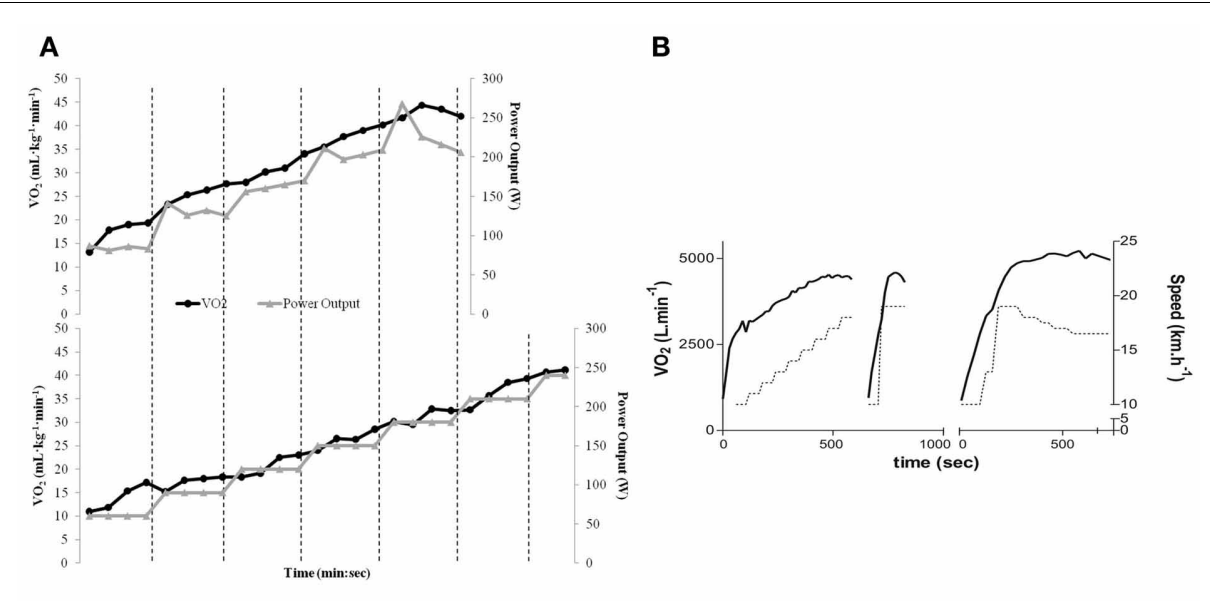

FIGURE 1 | (A) $\mathrm{VO}_{2}$ and power output data for the self-paced incremental protocol (top) and conventional incremental protocol (bottom) in a representative subject. A higher $\mathrm{VO}_{2}$ max (group mean $\approx 8 \%$ ) was achieved in the self-paced incremental protocol during submaximal workload. (B) $\mathrm{VO}_{2}$ and speed data for the conventional incremental test (left) + verification phase (middle) and for the decremental protocol (right) in a representative subject. A higher $\mathrm{VO}_{2}$ max (group mean $\approx 4.4 \%$ ) was achieved in the decremental protocol during submaximal workload. $\mathrm{VO}_{2}$ is represented by solid lines, and dotted lines represent speed. "Reproduced from Mauger and Sculthorpe (2012) and Beltrami et al. (2012) with permission from BMJ Publishing Group Ltd." levels over time). This decremental protocol started in the speed used during the "verification phase" of the incremental test, which means, $1 \mathrm{~km} \mathrm{~h}^{-1}$ faster than the last stage accomplished during the conventional exercise test. This intensity was kept for $60 \%$ of the individual time that subjects were able to tolerate during the "verification phase," with a subsequent reduction in speed of $1 \mathrm{~km} \mathrm{~h}^{-1}$ for $30 \mathrm{~s}$ and consecutive reductions of $0.5 \mathrm{~km} \mathrm{~h}^{-1}$, in which each stage was kept for 30, 45 , 60, 90, and $120 \mathrm{~s}$, respectively. Similarly to the maximal self-paced incremental test (Mauger and Sculthorpe, 2012), the decremental test proposed resulted in significantly higher $\mathrm{VO}_{2} \max (\approx 4.4 \%$; Figure 1B) when compared to the conventional incremental exercise test (Beltrami et al., 2012).

The main explanation suggested by the authors for the results found in the first study (Mauger and Sculthorpe, 2012) is that the nature of the selfpaced protocol may have allowed a higher power output for the same level of perception of effort or discomfort, leading to greater $\mathrm{VO}_{2}$ max before voluntary exhaustion. This occurred despite heart rate, ventilation, and respiratory exchange ratio values being similar to the conventional protocol. Additional suggestions as a greater relative contribution of oxygendependent type 1 fibers with a consequent reduction in the anaerobic component of the test, and/or an increase in the oxygen demand and utilization due to the high power output in the last stage of the self-paced incremental test, may also have contributed to the greater $\mathrm{VO}_{2}$ max found (Mauger and Sculthorpe, 2012). It is noteworthy that criticisms have already been raised to this study (Chidnok et al., 2013). At the same time the authors of the second study (Beltrami et al., 2012) suggest that differences in the anticipatory workload perception of the protocols, growing in the conventional incremental test and reducing in the decremental test, might have impacted the sympathetic or parasympathetic drives and led to different metabolic responses to exercise and to the greater $\mathrm{VO}_{2}$ max. Surprisingly, both studies showed that either untrained (Mauger and Sculthorpe, 2012), or trained (Beltrami et al., 2012) individuals attained the greater $\mathrm{VO}_{2} \max$ values during submaximal workloads, challenging the traditional concept that $\mathrm{VO}_{2} \max$ occurs at the maximal workload.

\section{IMPLICATIONS OF THE NEW FINDINGS}

Once recognized and further corroborated that current $\mathrm{VO}_{2}$ max measurement methods (i.e., conventional incremental exercise protocol) provide, in fact, submaximal values, which would be the implications of the new true $\mathrm{VO}_{2}$ max values found (Beltrami et al., 2012; Mauger and Sculthorpe, 2012) upon the existing body of knowledge relating to this area? In our opinion, a considerable portion of the scientific knowledge would be mildly affected, due to the existence of systematic error. For instance, studies aiming to verify the effect of specific interventions upon $\mathrm{VO}_{2}$ max already have $\mathrm{VO}_{2} \max$ underestimations aggregated into their results. As pre- and postintervention values are measured by the same protocol, the intervention effects upon $\mathrm{VO}_{2}$ max values would still be correctly measured, despite underestimation of $\mathrm{VO}_{2}$ max true value. In contrast, studies based upon $\mathrm{VO}_{2}$ max percentages, as the aerobic training zone for cardiorespiratory fitness, for example, which habitually varies around 50 and $85 \%$ of $\mathrm{VO}_{2}$ max, would have its interval range shifted to the right. Likewise, it would be necessary to review the indirect equations to estimate $\mathrm{VO}_{2} \mathrm{max}$, as they make use of $\mathrm{VO}_{2} \mathrm{max}$ reference values that are, according to the new findings (Beltrami et al., 2012; Mauger and Sculthorpe, 2012), submaximal. Nevertheless, knowing the underestimation magnitude of the $\mathrm{VO}_{2} \max$ by the conventional incremental protocols, mathematical equations would be able to provide a posteriori corrections, reducing/correcting such inaccuracies. 
Contrary to the relatively minor impact described above, the findings of greater $\mathrm{VO}_{2}$ max than the ones commonly found during conventional incremental exercise tests conflict with the theoretical models proposed to explain its limiting/regulatory mechanisms (Ekblom, 2009; Noakes and Marino, 2009). If the $\mathrm{VO}_{2} \max$ values found so far during conventional incremental tests are limited by the maximal capacity of the heart to provide $\mathrm{O}_{2}$ to the muscles (Ekblom, 2009), how can one explain such an increase (Beltrami et al., 2012; Mauger and Sculthorpe, 2012)? We identify two possibilities. The theoretical model may still be correct, that means, $\mathrm{VO}_{2}$ max is indeed limited by the maximal capacity of the heart, though, the $\mathrm{VO}_{2}$ max values found during conventional incremental tests are not truly maximal, and alternative protocols would be able to increase it. In opposition, the model may be wrong in stating that $\mathrm{VO}_{2} \mathrm{max}$ is primarily limited by the cardiac capacity, and another mechanism might exist to explain its limitation/regulation. The other theoretical model (Noakes and Marino, 2009), on its turn, also conflicts with the findings. If the brain regulates the number of motor unit recruited during exercise in order to prevent catastrophic failure in bodily systems, thus regulating the $\mathrm{VO}_{2}$ max achievable, why would the brain allow individuals during these two new protocols (Beltrami et al., 2012; Mauger and Sculthorpe, 2012) to attain $\mathrm{VO}_{2} \max$ values greater than during the conventional incremental tests? Would not the brain, based on afferent feedback from various systems, regulate the number of motor unit recruited in a similar fashion, independently of the exercise protocol performed?

A possible explanation for the recent findings may be found reaching back to the proposal by Jones and Killian (2000), who reviewed evidence to show that, rather than limitations based on the capacity of oxygen-delivery mechanisms, cardiorespiratory and exercise limitations are symptom-based. These authors, considering peripheral and central perceptions of effort data, raised the importance of considering these symptoms as limiting factors when measuring exercise performance and $\mathrm{VO}_{2} \max$ (Jones and Killian, 2000). A recent theoretical model further emphasizes the paramount importance of effort on endurance exercise performance regulation and tolerance (Marcora and Staiano, 2010; Smirmaul et al., 2013). The higher $\mathrm{VO}_{2}$ max values achieved (Beltrami et al., 2012; Mauger and Sculthorpe, 2012) may have been associated with altered perceptual responses due to the differences in the protocols used. However, this possibility remains speculative.

\section{CONCLUSION}

The proposals of different exercise protocols which result in greater $\mathrm{VO}_{2}$ max values than commonly found during the conventional incremental exercise tests should interest the exercise and sports physiology community. At the same time that such findings mildly impact a considerable portion of knowledge, they challenge, for instance, the theoretical models to explain $\mathrm{VO}_{2}$ max limitation/regulation. Still, they also challenge the concept that $\mathrm{VO}_{2} \mathrm{max}$ occurs at the maximal workload. While recent work has shown that it is possible to maintain a conventional $\mathrm{VO}_{2}$ max plateau up to $15 \mathrm{~min}$ by decreasing individuals' workload, that means, during submaximal work (Petot et al., 2012; Billat et al., 2013), it is unknown whether the same is possible for the superior $\mathrm{VO}_{2}$ max values found (Beltrami et al., 2012; Mauger and Sculthorpe, 2012). The suggestion that $\mathrm{VO}_{2} \max$ values are task-dependent, and that the conventional incremental exercise test does not produce true maximal values is attractive. However, understanding how these new exercise protocols produce higher $\mathrm{VO}_{2}$ max values, the influences of different protocols on perceptual responses and $\mathrm{VO}_{2}$ max measurement, determining its full implications and applications, and the specific limiting/regulatory mechanisms underpinning $\mathrm{VO}_{2}$ max, are new horizons that sports and exercise scientists may explore.

\section{REFERENCES}

Astorino, T. A. (2009). Alterations in VOmax and the VO plateau with manipulation of sampling interval. Clin. Physiol. Funct. Imaging 29, 60-67. doi: 10.1111/j.1475-097X.2008.00835.X

Astorino, T. A., Willey, J., Kinnahan, J., Larsson, S. M., Welch, H., and Dalleck, L. C. (2005). Elucidating determinants of the plateau in oxygen consumption at VO2max. Br. J. Sports Med. 39, 655-660. discussion: 660.

Bassett, D. R., and Howley, E. T. (2000). Limiting factors for maximum oxygen uptake and determinants of endurance performance. Med. Sci. Sports Exerc. 32, 70-84.

Beltrami, F. G., Froyd, C., Mauger, A. R., Metcalfe, A. J., Marino, F., and Noakes, T. D. (2012) Conventional testing methods produce submaximal values of maximum oxygen consumption. $\mathrm{Br}$. J. Sports Med. 46, 23-29. doi: 10.1136/bjsports2011-090306

Bergh, U., Ekblom, B., and Astrand, P. O. (2000). Maximal oxygen uptake "classical" versus "contemporary” viewpoints. Med. Sci. Sports Exerc. 32, 85-88.

Billat, V., Petot, H., Karp, J. R., Sarre, G., Morton, R. H., and Mille-Hamard, L. (2013). The sustainability of VO2max: effect of decreasing the workload. Eur. J. Appl. Physiol. 113, 385-394. doi: 10.1007/s00421-012-2424-7

Chidnok, W., Dimenna, F. J., Bailey, S. J., Burnley, M., Wilkerson, D. P., Vanhatalo, A., et al. (2013) $\mathrm{VO} 2 \mathrm{max}$ is not altered by self-pacing during incremental exercise: reply to the letter of Alexis, R. Mauger. Eur. J. Appl. Physiol. 113, 543-544. doi: 10.1007/s00421-012-2563-x

Day, J. R., Rossiter, H. B., Coats, E. M., Skasick, A., and Whipp, B. J. (2003). The maximally attainable VO2 during exercise in humans: the peak vs. maximum issue. J. Appl. Physiol. 95, 1901-1907.

Doherty, M., Nobbs, L., and Noakes, T. D. (2003). Low frequency of the "plateau phenomenon" during maximal exercise in elite British athletes. Eur. J. Appl. Physiol. 89, 619-623. doi: 10.1007/s00421003-0845-z

Ekblom, B. (2009). Counterpoint: maximal oxygen uptake is not limited by a central nervous system governor. J. Appl. Physiol. 106, 339-341. discussion: 341-342.

Hill, A. V., and Lupton, H. (1923). Muscular exercise, lactic acid, and the supply and utilization of oxygen. Q. J. Med. 16, 135-171. doi: 10.1093/qjmed/os-16.62.135

Jones, N. L., and Killian, K. J. (2000). Exercise limitation in health and disease. N. Engl. J. Med. 343, 632-641. doi: 10.1056/NEJM200008313430907

Levine, B. D. (2008). VO2max: what do we know, and what do we still need to know. J. Physiol. 586, 25-34. doi: 10.1113/jphysiol.2007. 147629

Mancini, D. M., Eisen, H., Kussmaul, W., Mull, R., Edmunds, L. H., and Wilson, J. R. (1991). Value of peak exercise oxygen consumption for optimal timing of cardiac transplantation in ambulatory patients with heart failure. Circulation 83, 778-786. doi: 10.1161/01.CIR.83.3.778

Marcora, S. M., and Staiano, W. (2010). The limit to exercise tolerance in humans: mind over muscle. Eur. J. Appl. Physiol. 109, 763-770. doi: 10.1007/s00421-010-1418-6

Mauger, A. R., and Sculthorpe, N. (2012). A new VO2max protocol allowing self-pacing in maximal incremental exercise. Br. J. Sports Med. 46, 59-63. doi: 10.1136/bjsports-2011090006

Midgley, A. W., Bentley, D. J., Luttikholt, H., McNaughton, L. R., and Millet, G. P. (2008). Challenging a dogma of exercise physiology: does an incremental exercise test for valid $\mathrm{VO} 2$ max determination really need to last between 8 and 12 minutes. Sports Med. 38, 441-447. doi: 10.2165/00007256-200838060-00001 
Midgley, A. W., and Carroll, S. (2009). Emergence of the verification phase procedure for confirming "true" $\operatorname{VO}(2 \max )$. Scand. J. Med. Sci. Sports 19, 313-322. doi: 10.1111/j.1600-0838.2009. 00898.x

Midgley, A. W., McNaughton, L. R., Polman, R., and Marchant, D. (2007). Criteria for determination of maximal oxygen uptake: a brief critique and recommendations for future research. Sports Med. 37, 1019-1028. doi: 10.2165/00007256200737120-00002

Noakes, T. D. (1998). Maximal oxygen uptake: "classical" versus "contemporary" viewpoints: a rebuttal. Med. Sci. Sports Exerc. 30, 1381-1398.

Noakes, T. D., and Marino, F. E. (2009). Point: maximal oxygen uptake is limited by a central nervous system governor. J. Appl. Physiol. 106, 338-339. discussion: 341 .
Petot, H., Meilland, R., Le Moyec, L., Mille-Hamard, L., and Billat, V. L. (2012). A new incremental test for $\mathrm{VO} 2 \mathrm{max}$ accurate measurement by increasing VO2max plateau duration, allowing the investigation of its limiting factors. Eur. J. Appl. Physiol. 112, 2267-2276. doi: 10.1007/s00421-011-2196-5

Poole, D. C., Wilkerson, D. P., and Jones, A. M. (2008). Validity of criteria for establishing maximal O2 uptake during ramp exercise tests. Eur. J. Appl. Physiol. 102, 403-410. doi: 10.1007/s00421-0070596-3

Smirmaul, B. P. C., Dantas, J. L., Nakamura, F. Y., Pereira, G. (2013). The psychobiological model: a new explanation to intensity regulation and (in)tolerance in endurance exercise. Rev. Bras. Educ. Fis. Esporte. 27, 333-340.

Spurway, N. C., Ekblom, B., Noakes, T. D., and Wagner, P. D. (2012). What limits $[\mathrm{V}(\cdot)] \mathrm{O}(2 \mathrm{max})$. A symposium held at the BASES Conference, 6
September 2010. J. Sports Sci. 30, 517-531. doi: 10.1080/02640414.2011.642809

Received: 28 June 2013; accepted: 18 July 2013; published online: 05 August 2013.

Citation: Smirmaul BPC, Bertucci DR and Teixeira IP (2013) Is the $\mathrm{VO}_{2}$ max that we measure really maximal? Front. Physiol. 4:203. doi: 10.3389/fphys.2013.00203

This article was submitted to Frontiers in Exercise Physiology, a specialty of Frontiers in Physiology.

Copyright (c) 2013 Smirmaul, Bertucci and Teixeira. This is an open-access article distributed under the terms of the Creative Commons Attribution License (CC BY). The use, distribution or reproduction in other forums is permitted, provided the original author(s) or licensor are credited and that the original publication in this journal is cited, in accordance with accepted academic practice. No use, distribution or reproduction is permitted which does not comply with these terms. 